\title{
sFEra APP: description and usability of a novel tablet application for executive functions training
}

Coricelli, C. ${ }^{1,2^{*}}$, Aiello, M. ${ }^{1 *}$, Lunardelli, A. ${ }^{3 \dagger}$, Galli, G. ${ }^{4 \dagger}$, Rumiati, R. I. ${ }^{1}$

${ }^{*}{ }^{\dagger}$ Equal contribution

${ }^{1}$ Area of Neuroscience, SISSA, Trieste, Italy

${ }^{2}$ University of Western Ontario, London, Canada

${ }^{3}$ Rehabilitation Division, Department of Integrated Neuroscience and Occupational Medicine, Azienda Sanitaria Universitaria Giuliano Isontina, Trieste Italy

${ }^{4}$ Neurology and Stroke Unit, ULSS4 Veneto Orientale, Portogruaro, Italy

\section{Corresponding author:}

Carol Coricelli, Department of Psychology, Western University, Western Interdisciplinary Research Building, 1151 Richmond St, London, ON N6A 3K7 (Canada), carolcoricelli@gmail.com

\begin{abstract}
Executive functions include functions such as planning, working memory, inhibition, mental flexibility, action monitoring and initiation, and are essential to carry out an independent everyday life. Individuals suffering from brain injury, such as a stroke, very commonly experience executive deficits that reduce the capacity to regain functional independence. In recent years, there has been a growing interest in developing tablet computer-based cognitive training programs for stroke patients. In this respect, we described and evaluated the usability of a novel tablet application (app) for executive function training, developed in the context of the MEMORI-net project, a cross-border Italy-Slovenia program for the rehabilitation of stroke patients. We conducted a pilot study with a non-clinical sample of 16 participants to obtain information about the usability of the app. Our descriptive analyses suggest that most users were satisfied with the overall experience and the app was highly usable, even with little previous experience with tablet applications. Acceptability and effectiveness will need to be evaluated in a clinical randomized controlled study.
\end{abstract}

\section{Keywords}




\section{INTRODUCTION}

Executive functions (EFs) refer to those processes which are crucial for goal-oriented behaviors which allow to successfully interact in the world (Gazzaniga, Ivry \& Mangun, 2006). Under this overarching definition, a heterogeneous group of mental capacities such as planning, working memory, inhibition, mental flexibility, and action monitoring and initiation fundamental for most of the daily actions is included (Damasio, 1995; Burgess et al., 2000; Chan et al., 2008 for a review) (See Figure 1).

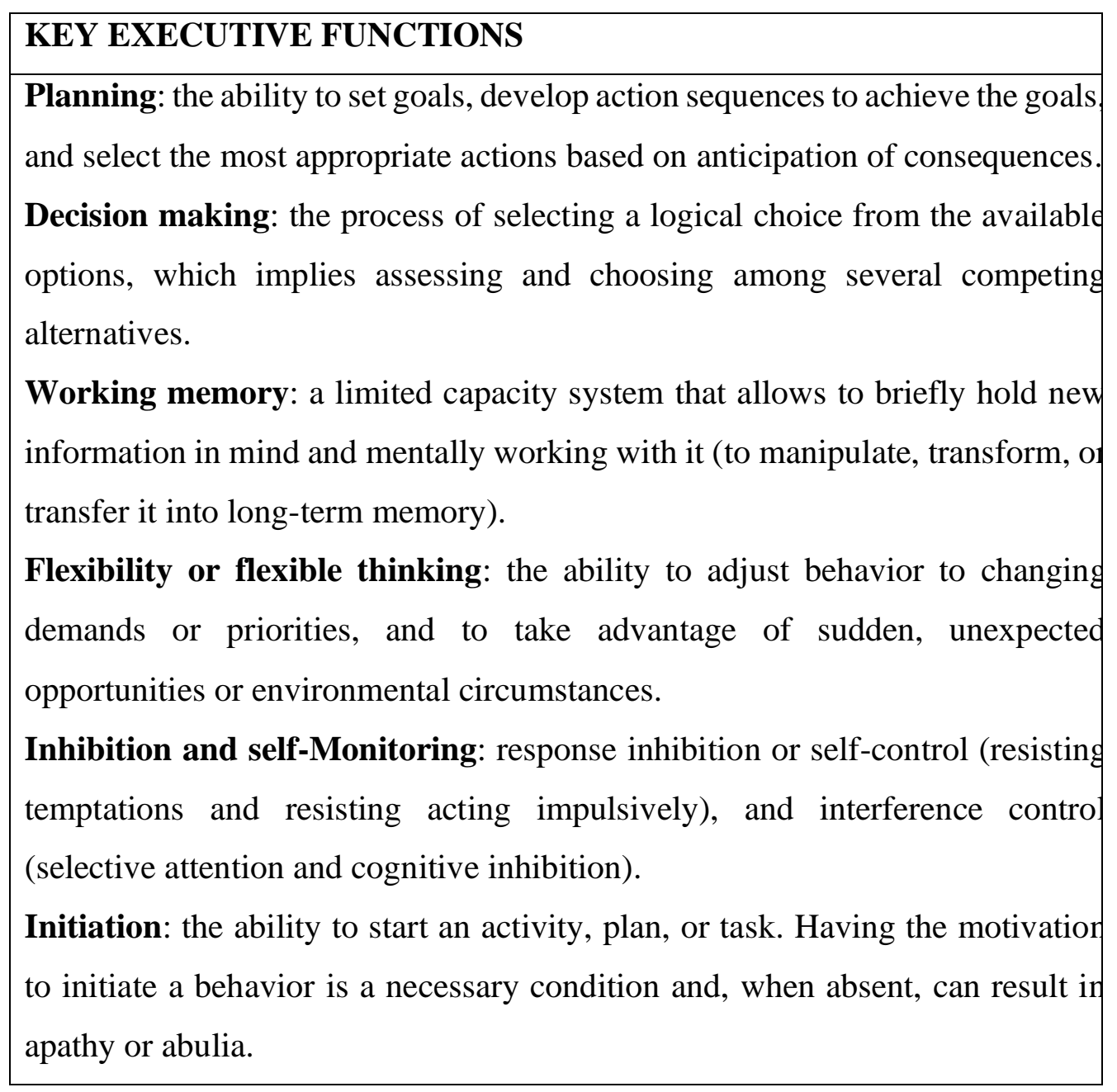

Figure 1. Description of the key executive functions (EFs) Anderson et al. (2002).

Indeed, the substantial increase in interest in EFs over the years is likely due to their pivotal role in everyday life (Vaughan \& Giovanello, 2010). EFs are critical to organizing our day (e.g. what has to be done, in which order, how long does it take, etc.) or when it is necessary to modify our plans due to 
changing contingencies or unexpected events, and unforeseen actions need to be taken. We routinely use EFs to learn new actions, make decisions and correct mistakes, engage in non-automatic routine behaviors that require constant monitoring, or complete complex or even dangerous behaviors. EFs are also essential to accurately evaluate and predict the success or failure of our behaviors: they are crucial to analyze the causes of mistakes and review the sequence of actions to plan a better strategy at the next occasion. A significant amount of flexibility is necessary to generate an alternative plan and switch from one coping behavior to another.

An impairment of such functions will necessarily lead to a dramatic impoverished everyday life by limiting the ability to adjust to environmental demands or changes. Disorders of attention-executive functioning are among the most frequent cognitive deficits in adults and they can be observed in aging, as well as following numerous neurological diseases, such as traumatic brain injury, stroke, multiple sclerosis, dementia, and Parkinson's disease. In particular, executive deficits occur in as much as $75 \%$ of stroke patients (Povroznik et al., 2018) and result in a worse functional outcome since they interfere with the process of rehabilitation and recovery (Lesniak et al., 2008; Jankowska et al, 2017).

Given the considerable impact of EFs impairment on functional outcomes, it is of primary importance to identify rehabilitation strategies that are effective in increasing and improving these fundamental capacities. Historically, restoration and compensation are the two distinct approaches that have been proposed to rehabilitate cognitive functions in brain-damaged patients (Mateer, 2005). Restorative interventions were usually offered in paper-and-pencil mode but, with the rapid growth and spreading of computer devices, computer-based training has gained popularity and nowadays represents an appealing alternative option (Sigmundsdottir, Longley, \& Tate, 2016). Indeed, it has several advantages over conventional training practices. Traditional cognitive programs usually require faceto-face contact, reaching the hospital or the therapist's practice, arranging schedules, and travel time; further, as they are carried out in one-to-one mode, they can be very expensive. Computer-assisted training instead can be carried out at any time and is cost-effective since people can do it at home; it can be self-paced and tailored to particular impairments and can provide immediate feedback regarding the success or failure of the exercises. Also, it consents the recording of patients' sessions, storing their results, as well as graphs of rehabilitation progress. With recent technological innovations, applications (apps) for mobile phones and tablets have quickly developed making cognitive training more attractive, stimulating and fun, all aspects that are important to promote neuroplasticity (Tacchino et al, 2015). Apps make also training sessions more feasible and flexible because they are user-friendly, and people can exercise wherever they want and at the most convenient time of the day. Currently, there are multiple apps for cognitive training that have been used in individuals with brain damage i.e. Cogmed (working memory training program, www.cogmed.uk.com), Luminosity (games-based brain training 
program, www.lumosity.com), Cognifit (neuropsychological tests and brain training program, Www.cognifit.com/it). These app-based games closely mimic traditional cognitive tasks, such as digit span and dual tasks. Their evidence for transfer effects on working memory, processing speed, and attention is strong (Harris et al., 2018; Melby-Lervåg and Hulme, 2013). However, to our knowledge, only a few of them have specifically focused on executive functions and have been developed to be used as a clinical tool with stroke patients, therefore the need to develop the present tablet application. In this article, we describe the initial usability testing of the sFEra APP, a novel tablet app-based cognitive training that is focused on attention-executive functioning. The rationale for designing a new APP was to develop a cognitive rehabilitation tool suitable for stroke patients. The APP has been developed in the context of the MEMORI-net program, a cross-border Italy-Slovenia project that aimed to delineate new common clinical standardized protocols for the rehabilitation of stroke patients. Testing the usability of the APP was necessary before a possible deployment during the rehabilitation of stroke patients involved in the program. For this reason, the digitized exercises were performed by a group of 16 healthy adults.

\section{MATERIALS AND METHODS}

\section{Participants}

This study involves adults who do not have cognitive impairment. For this reason, the following inclusion criteria were defined: (1) aged $\geq 45$ years and (2) a Montreal Cognitive Assessment score $\geq$ 21. In addition, they completed the Frontal Assessment Battery (FAB, Dubois, et al., 2000). All participants provided informed consent to take part in the study which was approved by SISSA's Ethics committee. The study conformed with the Declaration of Helsinki.

\section{sFEra APP}

\section{Overview}

sFEra is an Android-based app. The opening screen is shown in Figure 2. It includes five areas of exercises: (a) Attention, (b) Control and inhibition, (c) Working memory, (d) Planning, (e) Flexibility. Each area includes two exercises, and each exercise has 10 levels with incremental difficulties (except one of the exercises, see below). The app records each task session provides real-time feedback and keeps a record of subjects' performance. 


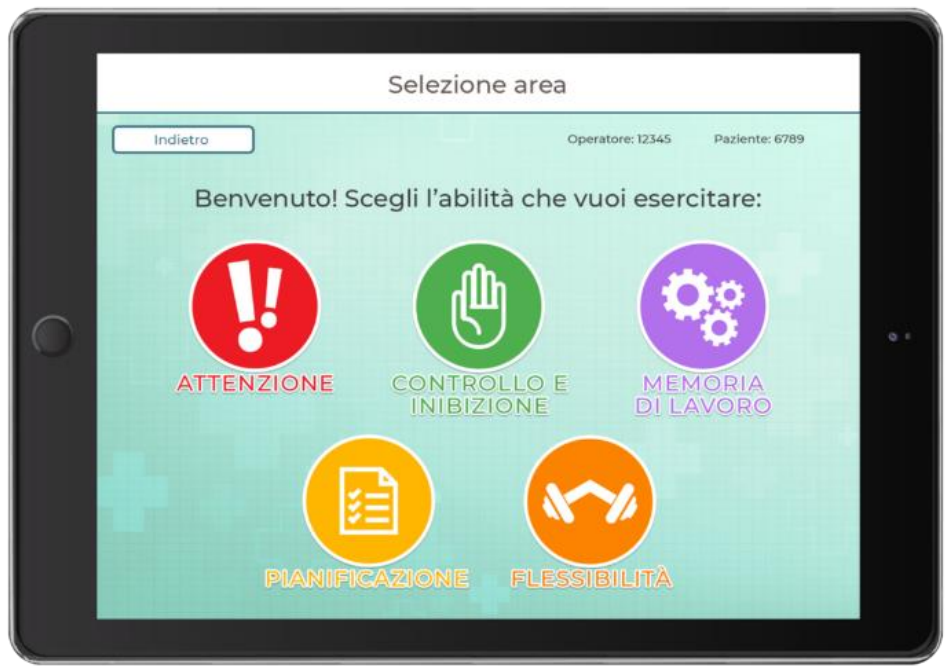

Figure 2. The opening screen of the sFEra app (Italian version).

(a) Attention. This section includes two exercises: 1- "Chi cerca trova" (a barrage task) and 2"L'imprevisto" (an oddball task)

\section{1- Barrage task}

This exercise was designed to evaluate and train participants' ability to direct their attention on the target items tuning out the non-relevant information (distractors).

The participants are instructed to search for the target items in a panel of distractors (see Figure 3). The distractors can change in both color and size of the item. Participants have to respond as quickly as possible. The following measures are recorded: total time of completion of each level (in milliseconds), number of correct targets selected (hits), number of targets selected more than once, number of distractors erroneously selected (false alarms). This exercise has 10 levels with incremental difficulties. Each level has three different scenarios (fruit, fishes, and cups) and three different backgrounds (easy, medium, hard). Different instructions characterize each level, the participant is instructed to select the target items based on color, shape, or size. Once participants select the item either a green tic (correct answer) or a red cross (incorrect answer) will appear on the item. Once the participant has pressed the end button on the screen, the level is considered completed and the participant will receive feedback with the score of the level. The participants will continue to the next level once $75 \%$ of correct answers are reached.

\section{2- Oddball task}

This exercise was designed to evaluate and train participants' sustained attention and vigilance.

The participants are instructed to avoid the target item (a hole in the street, see Figure 3) by pressing the $(\mathrm{x})$ button positioned below the screen, while an avatar is riding a bicycle through a street of a city. Other non-target distractors appear on the screen (puddle, manhole, a stack of leaves, wastepaper). The 
percentage of targets and distractors varies at each level, the target is always infrequent (max $15 \%$ of total trials) since it is aimed at testing participants' sustained attention and vigilance, the total time (in minutes) of each level also increases (range $=3-12$ minutes). The following measures are recorded: average time (in milliseconds) in response to the target stimuli, accuracy was quantified as the number of target stimuli detected, missed targets (omissions), and the total number of errors (responses to nontarget stimuli).

At the end of each level, the participants receive feedback on their performance based on their accuracy (total number of correct answers). The participants will continue to the next level once $75 \%$ of correct answers are reached.

(b) Control and inhibition. This section includes two exercises:1- "Alto là" (a Go/no go task)

\section{2- "Non farti distrarre" (a Stroop-like task)}

\section{1- Go/no go task}

This exercise was designed to evaluate and train participants' inhibitory control function since in the task they are forced to refrain from their actions. The participants are instructed to press the button positioned below each item (see Figure 3) as fast as they can only when the "go" stimuli are presented in the center of the screen and not to press for the "no-go" stimuli. Both, "go" and "no-go" stimuli changed in the different levels (i.e. simple geometric shapes, outline simple shapes, colored pictures). The chosen stimuli differed across the 10 levels but included colored geometrical shapes (i.e. square, triangle, circle, pentagon, see Figure 3), colored outline object figures (i.e. flower, tree, bell, book), colored outline animal figures (i.e., sheep, duck, pig, tortoise) and colored images depicting objects, animals, flowers and plants or foods. Throughout the levels, the participants had to respond to the color, shape, or identity of the stimuli. Each stimulus was presented at the center of the screen with an intertrial interval (ITI) randomly assigned between 500-1000ms the image remained on the screen for $1000 \mathrm{~ms}$. The following measures are recorded: total time (in milliseconds) to respond to each element of the Go- no go tasks (reaction times, RTs) and total time for each level (in seconds). RTs to each "go" item and the average for all of the "go" stimuli are registered. Number of correct responses to go stimuli (hits), number of incorrect responses to "no-go" stimuli (false alarms), number of missed responses to "go" stimuli (misses). After each level, the participant would receive feedback informing on the total number of correct answers. The participants will continue to the next level once $75 \%$ of correct answers are reached.

\section{2- Stroop-like task}

This exercise was designed to evaluate and train participant's ability to stay focused and inhibit distractions and interferences since participants had to actively ignore aspects of the stimuli that could 
lead to mistakes. This exercise had to versions each with 5 levels with incremental difficulties: a numerical one (2a. "Occhio ai numeri") and a verbal one (2b. "Occhio alle parole")

2a- Numerical Stroop-like task

We had participants respond to the bigger (or smaller depending on the block) number with bigger meaning written in a larger font regardless of the quantity, therefore participants had to ignore the actual numerical value of the number since it could distract them and lead to errors (see Figure 3). The following measures are recorded: accuracy (\% of correct answers) and reaction times (RTs in milliseconds to each stimulus and average RTs in milliseconds for each block).

\section{2b- Verbal Stroop-like task}

Participants had to respond with the button "big" to a word written in upper case and "small" to a word written in lower case (regardless of the meaning of the word, i.e. giant, dwarf, big, SMALL). Participants had to ignore the meaning of the word since it could distract them and lead to errors. The following measures are recorded: accuracy (\% of correct answers) and reaction times (RTs in milliseconds to each stimulus and average RTs in milliseconds for each block).

(c) Working Memory. This section includes two exercises. 1-“Tienimi a mente” (a running span Task) and 2-“Occhio alla regola" (an information manipulation task)

\section{1- Running Span task}

This exercise was designed to evaluate and train participants' working memory span, the numbers (or letters) that they can keep in mind. Strings of elements (numbers or letters) would visually appear on the center of the screen ( 1 item at the time, see Figure 3 ) in the end a keyboard would appear, and participants had to select from memory elements depending on the instructions. The number of elements of the string and the number of elements to recall increased across levels, initially participants were presented with strings of 2 and 3 elements and had to recall the last element, the elements increased to 4 and participants had to recall the last 2 elements, in the final level the strings were long up to 10 elements and participants had to recall the last 3 elements. Presentation time (initially 2 s) decreases to $1 \mathrm{~s}$ with increasing levels. Each level lasted 120 seconds, after each level the participant receives feedback on the performance. The following measures are recorded: accuracy (number of recalled elements, both raw score, and percentage of correct answers) the total time (in milliseconds) to recall each sequence, and total time (in seconds) to complete the level. The participants will continue to the next level once $75 \%$ of correct answers are reached.

\section{2- Information Manipulation task}

This exercise was designed to evaluate and train participants' working memory abilities to update different rules they had to keep in mind. Stimuli were presented in the auditory modality in this 
exercise. The participant listens to a sequence of numbers (or letters) and has to respond on a keyboard ordering them following the rule of the instruction, following some examples of rules: order first odd and then even numbers in a sequence of numbers, first consonants, and then vowels in a sequence of letters, ordering numbers presented with a random order in increasing order from the smallest to the largest, ordering letters presented in a random order alphabetically. The following measures are recorded: accuracy (number of recalled elements, both raw score, and percentage of correct answers) the total time (in milliseconds) to recall each sequence, and total time (in seconds) to complete the level. The participants will continue to the next level once $75 \%$ of correct answers are reached.

(d) Planning. This section includes two exercises:1-"Passo dopo passo" (an action sequences task) 2"Pianifica le tue mosse" (a planning task)

\section{1- Action Sequences task}

This task was designed to evaluate and train participants' ability to order action sequences, starting from simple common actions requiring few steps to more complicated and elaborate ones. Stimuli were presented on the screen as boxes on the left side of the screen containing sentences that the participant had to order, by moving them to the boxes on the right of the screen, according to the order of actions required to complete the sequence (see Figure 3). Simple actions including 3 steps were for example "Drink: 1) take the bottle, 2) open the bottle and 3) drink from the bottle", "Brush your teeth: 1) take the toothbrush, 2) put the toothpaste, and 3) brush my teeth", more complex action sequences including 5 actions were for example "Planting a plant: 1) take a vase, 2) put the soil in the vase, 3) plant the seed, 4) water the soil and 5) put the vase in the sun", sequences on level 10 reached 10 steps of unusual actions like "changing the tire of a bicycle". The following measures are recorded: accuracy (number sentences in the correct order). Participants received feedback after each sequence with the number of correct answers. The participants will continue to the next level once all sentences describing the action are correctly ordered.

\section{2- Planning task}

This task was designed to evaluate and train participants' ability to strategically plan actions to find the way to solve the task since once the action began one could not go back. The exercise required the participant to collect shells presented on a grid representing a net (see Figure 3), to move along the grid there were specific rules: at each intersection of the grid the subject can move left or right but cannot go backward, the subject had to follow the lines of the grid. Additional rules, based on the color of the shells, were added moving up with the harder levels, from level 4 there was a 1-minute limit to complete the task. The following measures are recorded: total time (in milliseconds) to complete each exercise 
and total time for each level (in seconds). The participants will continue to the next level once 100\% of correct answers are reached in the two exercises of the level.

(e) Flexibility. This section includes two exercises:1- "Pronti a cambiare" (switching task), 2-"2 in 1" (dual-task)

\section{1- Number/letter Switching task}

This task was adapted from Rogers and Monsell (1995) and was designed to evaluate and train participants' ability to change their responses based on quick and variable instructions. The participants were presented with a rectangle divided into 4 quadrants (top left, top right, bottom left, bottom right), in the rectangle, a couple of elements composed by a letter and a number could appear on each of the 4 quadrants when a couple of elements (i.e. N2) was presented in the top quadrants (both left and right) the subject was instructed to respond only to the number element, on the other hand when a couple of elements was presented in the bottom quadrants (both left and right) the subject was instructed to respond only to the letter element. Depending on the level the subject had to answer based on the properties of the elements such as is it a vowel or consonant letter, odd or even number, keeping in mind the quadrant rule, which was also present on the screen for the whole time of the exercise, the subject provided yes and no answers through a keypad on the screen.

The following measures are recorded: total time (in milliseconds) to respond to each element and total time for each level (in seconds), accuracy (number of correct answers, raw value, and \% of correct answers). The participants will continue to the next level once $75 \%$ of correct answers are reached in the two exercises of the level.

\section{2- Dual-task}

This task was designed to evaluate and train participants' ability to perform two tasks at the same time. The first task was the visual Go-no go task (Exercise b1) described above with the same characteristics and same stimuli, and the second task was an auditory Go-no go task but with sounds as stimuli instead of figures/pictures. The instructions regarding the visual Go-no go could include rules based on the color, shape, or identity of the stimuli and for the auditory Go-no go the subjects had to respond to the sound presenting a certain pitch (low or high depending on the level) and refrain from answering to the other pitch, both sounds were presented at the beginning to the subject to familiarize with them.

The following measures are recorded: total time (in milliseconds) to respond to each element (separate visual and auditory Go- no go tasks, reaction times RTs) and total time for each level (in seconds), accuracy (number of correct answers, raw value, and \% of correct answers). RTs to each "go" item and the average for all of the "go" stimuli are registered. Number of correct responses to go stimuli (hits), number of incorrect responses to "no-go" stimuli (false alarms), number of missed responses to "go" 
stimuli (misses). After each level, the subject would receive feedback informing on the total number of correct answers. The participants will continue to the next level once the $75 \%$ of correct answers is reached.
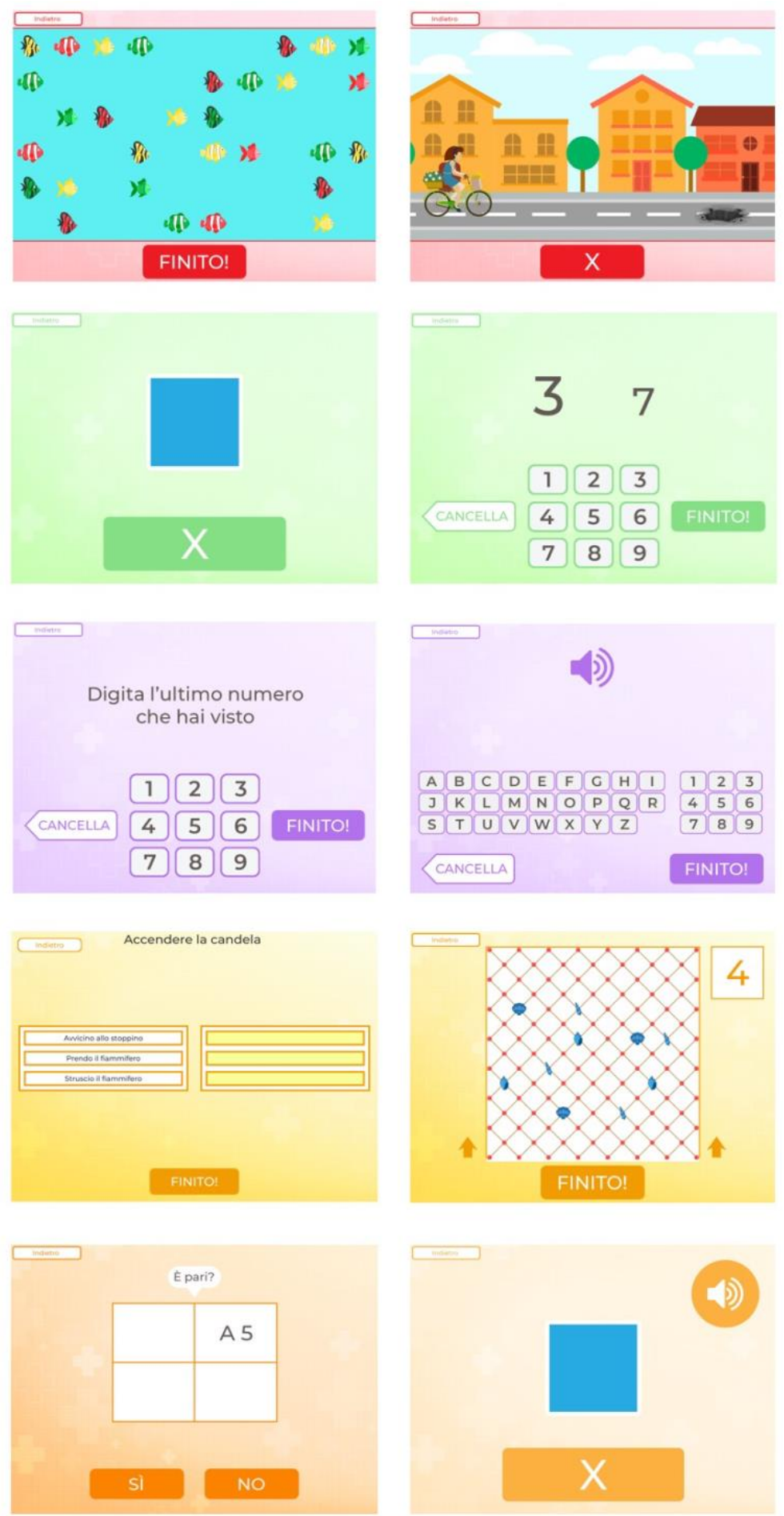

Figure 3. Examples of the exercises.

Participants in the study performed only two levels for each exercise. After, they completed the Usability questionnaire and a Questionnaire on experience and use of technology items. 


\section{Usability Questionnaire}

At the end of each exercise, participants had to respond to the following questions on a 5-point Likert scale (ranging 1-5; in brackets the extremes of the scale).

QA: "How would you judge the clarity of the instructions?" ([1] "very poor" - [5] "very good")

QB: "How would you judge the difficulty of the exercise?" ([1] "very low" - [5] "very high")

QC: "How would you judge the degree of satisfaction during the execution of the exercise?" ([1] "very poor" - [5] "very good")

Moreover, at the end of all of the exercises, the participant had to respond to the following questions referring to the whole sFEra APP on a 5-point Likert scale (ranging 1-5; in brackets the extremes of the scale).

Q1: "Overall how would you judge the clarity of the instructions of the app?" ([1] "very poor" - [5] "very good")

Q2: "How would you judge your interest in the app exercises?" ([1] "very poor" - [5] "very good")

Q3: "How would you judge your motivation to use again the app?" ([1] "very poor" - [5] "very good")

Q4: "How would you judge the graphics of the app?" ([1] "very poor" - [5] "very good")

Q5: "How would you judge your motivation while you executed the exercises?" ([1] "very poor" - [5] "very good")

Q6: "How would you judge your stress level while you executed the exercises?" ([1] "very poor" - [5] "very good")

Q7: "How would you judge your boredom level while you executed the exercises?" ([1] "very poor" [5] "very good")

Q8: "How would you judge your entertainment level while you executed the exercises?" ([1] "very poor" - [5] "very good")

Q9: "How useful would you judge the execution of the exercises?" ([1] "very poor" - [5] "very good")

\section{Questionnaire on experience and use of technology items}

Participants had to respond also to the following questions assessing their experience and hours per week to 3 different technology items (personal computer, tablet, and smartphone). Responses were provided on a 5-point Likert Scale (ranging 1-5; in brackets the extremes of the scale).

Q1: "How would you judge your experience with ... (personal computer/tablet/smartphone)" ([1] "very poor" - [5] "very good")

Q2: "On average how many hours per week do you use ... (personal computer/tablet/smartphone)" ([1] "less than 1 hour" - "more than 15 hours" [5]) 
Q3: "Did you have previous experience with cognitive training applications/exercises in the past" ([1] "never" - "very often" [5])

\section{RESULTS}

\section{Participants}

Sixteen subjects (11 females) took part in the pilot study, participants mean age was 59 years old (SD $=8.04$, range $=48-76)$, their mean years of education was 13.12 years $(\mathrm{SD}=3.70$, range $=5-19)$. All participants were Italian native speakers, did not have any neurodegenerative diseases nor peripheral motor deficits. Participants mean raw score on the MoCA scale was 28.12 ( $\mathrm{SD}=2.03$, range $=23-30)$. Participants mean raw score on the FAB scale was $17.87(\mathrm{SD}=0.34$, range $=17-18)$.

\section{Usability Questionnaire}

The questionnaire showed positive results. Most users were pleased with the overall experience. Specifically, $68.8 \%$ judged the experience as entertaining (Q8 of the Usability questionnaire, the percentage calculated considering scores 4 and 5; overall mean=3.6) and $68.8 \%$ as useful (Q9, mean=3.7). Moreover, $81.3 \%$ found that the graphics of the app has a high quality (Q4, overall mean=4.3), 56.3\% found the exercises of the app interesting (Q2, overall mean=3.8). Also, 81.25\% of them felt highly motivated during the execution of the task $(Q 5$, overall mean=4.3). All experienced low levels of stress (Q6, overall mean=2.5) and were not bored (87.5, Q7, overall mean=2). Importantly, the instructions of the app were easy to understand by $81.5 \%$ of the users $(\mathrm{Q} 1$, overall mean=4). Finally, users felt on average motivated to use the app again $(37.5 \%, \mathrm{Q}$, overall mean=2.93), see Figure 4 for the complete distribution of the responses for each question. 


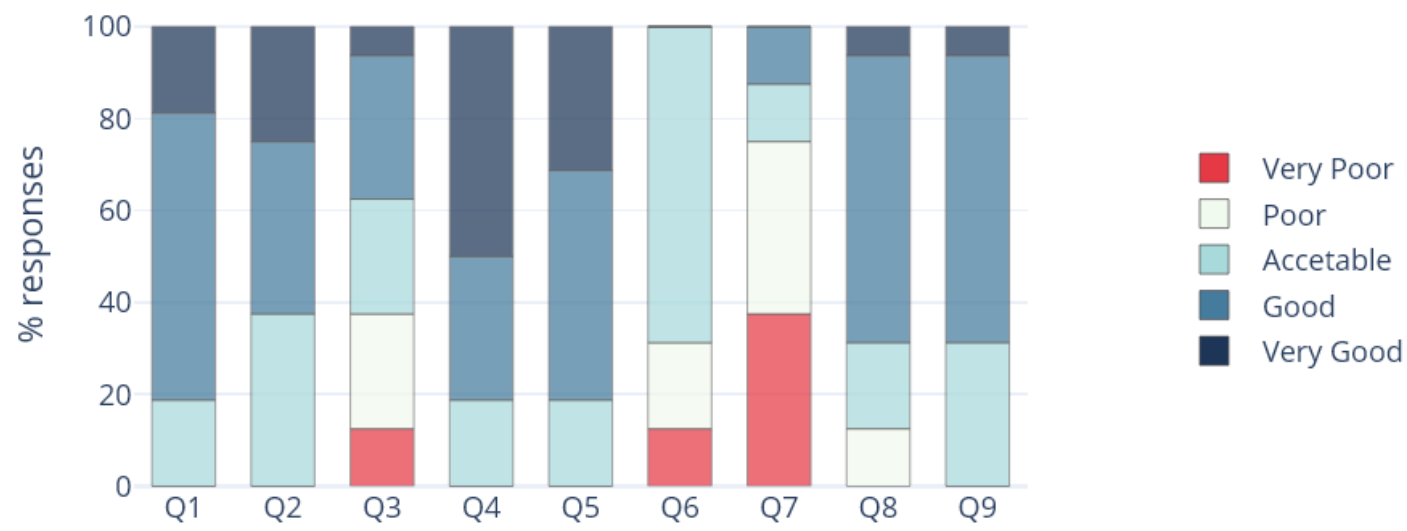

Figure 4. Overall usability experience. Q1= how would you judge the clarity of the instructions of the app; Q2 = did you find the exercises of the app interesting; Q3= Are you motivated to use again the app?; Q4="How would you judge the graphics of the app?"; Q5=“ How would you judge your motivation while you executed the exercises?"; Q6= How would you judge your stress level while you executed the exercises?"; Q7= How would you judge your boredom level while you executed the exercises?"; Q8= How would you judge your entertainment level while you executed the exercises; $Q 9=$ How useful would you judge the execution of the exercises?

For what concerns the single exercises, the average evaluations concerning the clarity of the instructions were in the range of 2.9 to 4.6 , which corresponds to a moderate to a very good rating. The exercise receiving the lowest mean ranking was the manipulation task ("Occhio alla regola"). This task was designed to train participants' working memory abilities to update different rules they had to keep in mind. For what concerns the difficulty of the exercises, the average evaluations were in the range of 1.5 to 3.3 indicating that they were perceived as low or moderately difficult. The exercise with the highest mean ranking was the planning task ("Pianifica le tue mosse"). This task was designed to train participants' ability to strategically plan actions to find the way to solve the task. Finally, the average evaluations concerning the level of satisfaction for each exercise were in the range of 3 to 3.8 which corresponds to a moderate to a good rating. The exercise with the highest satisfaction score was the barrage task ("Chi cerca trova"), while the exercise with the lowest score was the oddball task ("L’imprevisto"), see Figure 5 for the complete scores for each exercise. 

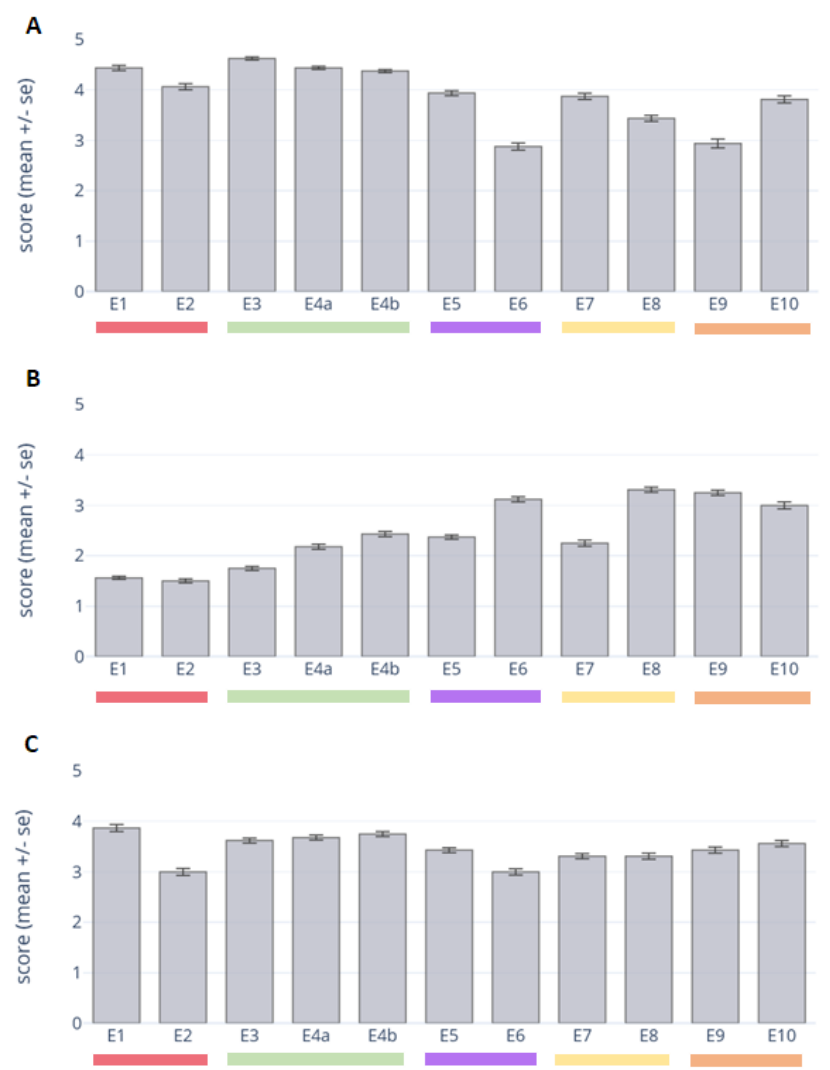

Figure 5. Individual exercises usability experience. A. Clarity of the instructions; B. Difficulty of each exercise; C. Satisfaction during the exercise execution. E1=Barrage task; E2= Oddball task; E3= Go/no go task; E4a= Modified Stroop task; E4b= Modified Stroop task E5= Digit span Task; E6= Manipulation task; E7=Planning $\mathrm{E} 8=$ Action sequences E9=Dual task E10=Switching.

\section{Questionnaire on experience and use of technology items}

Although most of the participants were familiar in using computer (mean $=3.19 \mathrm{SD}=1.16$ ), tablet $($ mean $=2.56 \mathrm{SD}=1.31)$ and smartphone $($ mean $=3.44 \mathrm{SD}=1.03)$ their previous experience with tablet applications was low (mean $=1.12 \mathrm{SD}=0.34)$ and they report to spend relatively little time in their use. Participants reported spending on average 2.75 hours per week on the personal computer ( $\mathrm{SD}=1.57)$, 1.87 hours per week on the tablet ( $\mathrm{SD}=1.26), 2.65$ hours per week on the smartphone ( $\mathrm{SD}=1.50$ ). Pearson's correlations between previous experience with tablets and the usability questionnaire were performed (see Table 1), resulting in a positive correlation between previous experience with tablets and the overall entertainment of the $\operatorname{app}(\mathrm{r}(14)=.53, \mathrm{p}=0.04)$. Interestingly, previous experience with tablets did not correlate with the clarity of the instructions, the motivation to perform the exercises, and the stress level. A second Pearson's correlation was performed between previous experience with brain training applications and the usability questionnaire (see Table 2), resulting in a positive correlation between previous experience with brain training applications and the overall willingness to use the 
application again $(\mathrm{r}(14)=.52, \mathrm{p}=0.04)$. Again, previous experience with brain training apps did not correlate with any other question of the usability questionnaire.

\begin{tabular}{|l|l|}
\hline & Experience tablet \\
\hline Q1 App Instructions & 0.08 \\
\hline Q2 App Interesting & 0.07 \\
\hline Q3 App Again & 0.41 \\
\hline Q4 App Graphics & 0.08 \\
\hline Q5 App Motivation & 0.27 \\
\hline Q6 App Stress & -0.07 \\
\hline Q7 App Boredom & -0.44 \\
\hline Q8 App Entertainment & $\mathbf{0 . 5 3 *}$ \\
\hline Q9 Useful & 0.37 \\
\hline
\end{tabular}

Table 1. Correlation of Experience with tablet and each question of the Usability questionnaire, significant results reported in bold.

\begin{tabular}{|l|l|}
\hline & $\begin{array}{l}\text { Experience brain training } \\
\text { applications }\end{array}$ \\
\hline Q1 App Instructions & -0.31 \\
\hline Q2 App Interesting & 0.06 \\
\hline Q3 App Again & $\mathbf{0 . 5 2} *$ \\
\hline Q4 App Graphics & 0.09 \\
\hline Q5 App Motivation & 0.2 \\
\hline Q6 App Stress & -0.03 \\
\hline Q7 App Boredom & -0.19 \\
\hline Q8 App Entertainment & 0.18 \\
\hline Q9 Useful & -0.17 \\
\hline
\end{tabular}

Table 2. Correlation of Experience with brain training applications and each question of the Usability questionnaire, significant results reported in bold. 


\section{DISCUSSION}

One in three people will experience a stroke, dementia, or both during their lives (Seshadri et al., 2007), and one in three individuals will sustain some degree of cognitive deficit after a stroke (Patel et al., 2003). Among them, visuospatial disorders and executive dysfunctions are those that are most commonly reported in association with poor functional assessments at admission and discharge. Specifically, individuals with executive deficits are those finding it harder to follow and benefit from the cognitive rehabilitation given their difficulty in initiating activities, maintaining a response, inhibiting action, and generalizing instructions to other tasks (Park et al., 2017). It is therefore mandatory to develop cognitive training specifically focused on these disorders, which may result effective and which may ensure a high adherence to the treatment and allow patients to perform everyday tasks involving planning, working memory, and more. Indeed, patients' withdrawal and boredom are quite common in conventional treatment programs, especially post-hospital discharge: as a result, many of them do not achieve the recommended intensity and duration of rehabilitation, thus reducing the clinical efficacy of protocols. Several factors may affect subjects' commitment therapy sessions: sessions are time-consuming, therapist-dependent, and imply travel costs. A growing body of research suggests that information and communication technologies (such as mobile apps) have an increasingly important role in the neuropsychological rehabilitation of patients with acquired brain injury (Gamito et al., 2015) and may help in overcoming such challenges. Indeed they have proved to be associated with high adherence to treatment in different contexts (see, for example, Arean et al., 2016) and individuals can potentially benefit from a longer duration of rehabilitation through the extension of therapeutic processes beyond the hospital, like patient's home. Here, we assessed the usability of a new tablet APP, the sFEra APP, that has been developed as a tool for the rehabilitation of executive functioning in stroke patients according to the MEMorinet program, a cross-border ItalySlovenia project that aims to delineate new common clinical standardized protocols for the rehabilitation of stroke. According to the international standard ISO 9241-11, usability can be defined as: "The extent to which a product can be used by specified users to achieve specified goals with effectiveness, efficiency, and satisfaction in a specified context of use". While the research related to usability has been the subject of extensive study, from a social inclusion point of view, more focus should be placed on the needs of aging adults (Klimova et al., 2018), as we prefigured in this pilot study. Results show that participants judged the use of the app as a positive experience and three themes emerged from usability testing:

(i) Clarity in the instructions, graphics, and content of the exercise obtained good ratings. Instructions appeared to be clear to the participants, overall, the app was scored 4 on a 5-point scale. Considering the single exercises, the clarity of instructions evaluations was in the range of 2.9 to 4.6 , which 
corresponds to a moderate to a very good rating. One of the most critical components of computerized task performance is clarity (Reppa and McDougall, 2015). The construct of clarity pertains to the transmission (or communication) of information, and the information quantity itself. Therefore, it includes the meaning of simplicity, as well. It also refers to how accessible and comprehensible the informational load of a given exercise is. Not only the clarity of instructions but also visual clarity plays an essential role in the user experience, as its impact is determined in the very first seconds of the interaction and determined by the sensory system of the individual (Bolte et al., 2015). Results from recent studies (see Lindgaard et al., 2006) suggest that aesthetics' judgment influences the totality of the subsequent experience. The same experiment also suggests that the time frame in which the first impressions form, can be as low as 50 milliseconds. Although they take place in a small amount of time compared to the total interaction, the immediate cognitive responses to the visual stimulation are necessary to the experience's evaluation.

(ii) Compliance was found to be high since participants resulted interested and motivated during task completion. This positive adherence seems to be triggered by the gamification process, a process where the user is rewarded upon achieving the goal of the app (Deterding et al, 2011). Different studies suggest that participants are pleased to be able to keep track of their progress and adherence to the treatment (Cheng et al., 2019, for a review). In this pilot test, a similar process is suggested by the positive correlation between experience with brain training applications and the willingness to use the application again, which is a promising aspect also for the continuation of the training after rehabilitation programs.

(iii) Importantly, it emerged that previous experience with tablets nor brain training apps was correlated with the usability questions, meaning that individuals without experience could clearly understand the tasks and perform them with enjoyment and motivation, without stress or boredom.

Nonadherence to clinical treatments is an important issue that needs to be addressed among rehabilitation programs, as it is partially responsible for a good rehabilitation outcome (Agency for Healthcare Research and Quality, 2016). Nonadherence to protocols not only affects patient clinical health outcomes but also patient quality of life and health care costs (World Health Organization, 2003) Mobile apps have the potential to improve adherence and management of treatments (Kaushal and Bates, 2002). The Unified Theory of Acceptance and Use of Technology (Venkatesh et al., 2003; Venkatesh et al., 2012) affirms that behavioral intention is the strongest predictor of technology use. Altogether, results suggest that the sFEra APP is highly usable and motivating, and as such can be proposed for cognitive rehabilitation interventions. Nevertheless, future investigations should test the acceptability of the app on a cohort of stroke patients and the effectiveness of the app in improving executive deficits and functional outcomes through a randomized controlled study, as well as 
measuring the cost-effectiveness of the app and its potential use in clinical practice. Several qualitative studies (see, e.g., Carabeo et al, 2014; White et al, 2015) support the implementation of computer devices during stroke recovery and demonstrate high acceptance and satisfaction of iPad-based rehabilitation programs. Tablets are considered easy to use, engaging and beneficial (White et al, 2015) in addition, tablet-based rehabilitation seems to be even preferred over conventional therapy (Carabeo et al, 2014). However, it is not yet clear whether tablet computerized brain-training programs can improve attention and executive functions. While many studies have shown positive results in healthy adults, (e.g., Anguera et al., 2013; Corbett et al., 2015) there is concern that improvements could be related to enhanced skills at using the apps rather than an actual improvement in cognition since marginal transfer effects to daily activities have been found (Owen et al., 2010; Gajewsky et al, 2020). The feasibility of the App should also be explored with stroke survivors to identify motor or communicative barriers that might prevent its adoption and utilization because of motor or speech/language impairments.

\section{CONCLUSIONS}

The results of this pilot study indicate that sFEra APP is a usable app and pave the way for future investigations to confirm its clinical validity. Research on mobile apps is necessary since this technology can augment the effects of face-to-face therapy and provide the opportunity for individuals to engage in homework tasks. This aspect is even more important in the light of the recent COVID-19 pandemic which has heightened the need for virtual cognitive assessment and training. We interpret the positive feedback on user experience as evidence that the sFEra APP is highly functional, motivating, readily accepted, and, as such, has the potential to represent an attractive tool for cognitive improvement and enhance therapy adherence. We also expect that the multidomain cognitive training of the App will greater enhance executive functioning and promote wider generalization to real-life than single cognitive process protocols.

\section{ACKNOWLEDGMENTS}

The present study was financed by the Interreg V-A Italia-Slovenia 2014-2020 program MEMORInet. We thank Enrico Tongiorgi for coordinating the program MEMORI-net and Francesco Darek Costa for helping with data collection.

\section{CONFLICT OF INTEREST}

The authors declare no competing financial interests. 


\section{DATA ACCESSIBILITY}

The data of the present study can be requested to the corresponding author.

\section{AUTHORS CONTRIBUTIONS}

CC, MA, AL, GG conceived the original idea of the present study and developed the exercises of the application which was realized by PIKKART Srl. CC and FDC collected the data for the present study under the supervision of MA. CC and MA performed the statistical analysis, CC, MA, AL, GG and RIR contributed in interpreting the results. CC, MA, AL, GG and RIR wrote the manuscript, reviewed and finalized the manuscript.

\section{REFERENCES}

Agency for Healthcare Research and Quality US (2016). Patient Safety in Ambulatory Settings. Rockville

Anderson, V., Levin, H. S., \& Jacobs, R. (2002). Executive functions after frontal lobe injury: A developmental perspective.

Anguera J., Boccanfuso J., Rintoul, J. Al-Hashimi O., Faraji F., Janowich J., Kong E., Larraburo Y., Rolle C., Johnston E. \& Gazzaley A. (2013).Video game training enhances cognitive control in older adults. Nature 501, 97-101.

Arean, P. A., Hallgren, K. A., Jordan, J. T., Gazzaley, A., Atkins, D. C., Heagerty, P. J., \& Anguera, J. A. (2016). The use and effectiveness of mobile apps for depression: results from a fully remote clinical trial. Journal of medical Internet research, 18(12), e330.

Appollonio I, Leone M, Isella V, Piamarta F, Consoli T, Villa ML, Forapani E, Russo A, Nichelli P. (2005). The Frontal Assessment Battery (FAB): normative values in an Italian population sample. Neurol Sci. 26(2):108-16.

Bölte, J., Hösker, T. M., Hirschfeld, G., \& Thielsch, M. T. (2017). Electrophysiological correlates of aesthetic processing of webpages: a comparison of experts and laypersons. PeerJ, 5 
Burgess, P. W., Veitch, E., de lacy Costello, A., \& Shallice, T. (2000). The cognitive and neuroanatomical correlates of multi-tasking. Neuropsychologia, 38, 848-863.

Carabeo C. G. G., Dalida C. M. M., Padilla E. M. Z, Rodrigo M. M. T. (2014). Stroke patient rehabilitation: A pilot study of an android-based game. Simulation \& Gaming, 45, 151-166.

Chan, R. C., Shum, D., Toulopoulou, T., \& Chen, E. Y. (2008). Assessment of executive functions: Review of instruments and identification of critical issues. Archives of clinical neuropsychology, 23(2), 201-216.

Cheng VWS, Davenport T, Johnson D, Vella K, Hickie IB. Gamification in Apps and Technologies for Improving Mental Health and Well-Being: Systematic Review. (2019). JMIR Ment Health. 26;6(6):e13717.

Conti, S., Bonazzi, S., Laiacona, M., Masina, M., \& Coralli, M. V. (2015). Montreal Cognitive Assessment (MoCA)-Italian version: regression-based norms and equivalent scores. Neurological Sciences, 36(2), 209-214.

Corbett A, Owen A, Hampshire A, Grahn J, Stenton R, Dajani S, Burns A, Howard R, Williams N, Williams G, Ballard C. (2015). The Effect of an Online Cognitive Training Package in Healthy Older Adults: An Online Randomized Controlled Trial. Journal of the American Medical Directors Association,.1;16(11):990-7.

Damasio, A. R. (1995). Toward a neurobiology of emotion and feeling: Operational concepts and hypotheses. The Neuroscientist, 1(1), 19-25.

Deterding S, Dixon D, Khaled R, Nacke L. (2011). From game design elements to gamefulness: defining. Proceedings of the 15th International Academic MindTrek Conference: Envisioning Future Media Environments; September 28-30, 2011; Tampere, Finland.

Gamito P, Oliveira J, Coelho C, Morais D, Lopes P, Pacheco J, Brito R, Soares F, Santos N, Barata AF. (2015). Cognitive training on stroke patients via virtual reality-based serious games. Disabil Rehabil.;39(4):385-388. 
Gajewski, P. D., Thönes, S., Falkenstein, M., Wascher, E., \& Getzmann, S. (2020). Multidomain Cognitive Training Transfers to Attentional and Executive Functions in Healthy Older Adults. Frontiers in Human Neuroscience, 14, 586963. https://doi.org/10.3389/fnhum.2020.586963

Gazzaniga, M. S., Ivry, R. B., \& Mangun, G. R. (2006). Cognitive Neuroscience. The biology of the mind. Norton: New York.

Harris DJ, Wilson MR and Vine SJ (2018) A Systematic Review of Commercial Cognitive Training Devices: Implications for Use in Sport. Front. Psychol.

ISO 9241-11:1998 Ergonomic requirements for office work with visual display terminals (VDTs) Part 11: Guidance on usability. 1998. [2019-11-21]. ISO: International Organization for Standardization

Jankowska, A. M., Klimkiewicz, R., Kubsik, A., Klimkiewicz, P., Śmigielski, J., \& WoldańskaOkońska, M. (2017). Location of the ischemic focus in rehabilitated stroke patients with impairment of executive functions. Advances in clinical and experimental medicine: official organ Wroclaw Medical University, 26(5), 767-776.

Kaushal R, Bates D. (2002). Information technology and medication safety: what is the benefit? Qual Saf Health Care. 11(3):261-5.

Klimova B and Valis M (2018) Smartphone Applications Can Serve as Effective Cognitive Training Tools in Healthy Aging. Front. Aging Neurosci. 9:436.

Leśniak, M., Bak, T., Czepiel, W., Seniów, J., \& Członkowska, A. (2008). Frequency and prognostic value of cognitive disorders in stroke patients. Dementia and geriatric cognitive disorders, 26(4), 356363.

Mateer, C. A. (2005). Fundamentals of cognitive rehabilitation. In P. W. Halligan \& D. T. Wade (Eds.) Effectiveness of Rehabilitation for Cognitive Deficits, 21-30. New York, NY: Oxford University Press.

Melby-Lervåg,M., and Hulme, C. (2013). Is working memory training effective? A meta- analytic review. Dev. Psychol. 49, 270-291. 
Owen A., Hampshire A., Grahn J. Stenton R., Dajani S., Burns A. S., Howard R. J. \& Ballard C. G. (2010). Putting brain training to the test. Nature 465, 775-778

Park, S. H., Sohn, M. K., Jee, S., \& Yang, S. S. (2017). The characteristics of cognitive impairment and their effects on functional outcome after inpatient rehabilitation in subacute stroke patients. Annals of rehabilitation medicine, 41(5), 734.

Patel, M., Coshall, C., Rudd, A. G., \& Wolfe, C. D. (2003). Natural history of cognitive impairment after stroke and factors associated with its recovery. Clinical rehabilitation, 17(2), 158-166.

Povroznik, J. M., Ozga, J. E., Haar, C. V., \& Engler-Chiurazzi, E. B. (2018). Executive (dys) function after stroke: special considerations for behavioral pharmacology. Behavioural pharmacology, 29(7), 638.

Reppa I, McDougall S. (2015). When the going gets tough the beautiful get going: aesthetic appeal facilitates task performance. Psychon Bull Rev. Oct;22(5):1243-54.

Rogers RD, Monsell S. (1995). Costs of a predictable switch between simple cognitive tasks. Journal of Experimental Psychology: General;124:207-231

Seshadri, S., \& Wolf, P. A. (2007). Lifetime risk of stroke and dementia: current concepts, and estimates from the Framingham Study. The Lancet Neurology, 6(12), 1106-1114.

Sigmundsdottir, L., Longley, W. A., \& Tate, R. L. (2016). Computerised cognitive training in acquired brain injury: A systematic review of outcomes using the international classification of functioning (ICF). Neuropsychological Rehabilitation, 26(5-6), 673-741.

Tacchino, A., Pedullà, L., Bonzano, L., Vassallo, C., Battaglia, M. A., Mancardi, G., ... \& Brichetto, G. (2015). A new app for at-home cognitive training: description and pilot testing on patients with multiple sclerosis. JMIR mHealth and uHealth, 3(3), e85.

Vaughan, L., \& Giovanello, K. (2010). Executive function in daily life: Age-related influences of executive processes on instrumental activities of daily living. Psychology and aging, 25(2), 343. 
Venkatesh V, Morris MG, Davis FD, Davis GB. (2003). User acceptance of information technology: toward a unified view. MIS $Q: 425-478$.

Venkatesh V, Thong J, Xu X. (2012). Consumer acceptance and use of information technology: extending the unified theory of acceptance and use of technology. MIS Q:157-178.

White J., Janssen H., Jordan, L., Pollack M. (2015). Tablet technology during stroke recovery: A survivor's perspective. Disability and Rehabilitation, 37, 1186-1192.

World Health Organization (2003). Adherence to Long Term Therapies evidence Actions. Switzerland:2003. 